\title{
EL HUMOR COMO HECHO PRAGMÁTICO EN ESPAÑOL
}

\author{
LEONOR RUIZ GURILlO \\ Universidad de Alicante. Grupo GRIALE \\ leonor.ruiz@ua.es
}

\begin{abstract}
Resumen: En este artículo se analiza la habilidad metapragmática del humor en español. Considerando que se trata de un hecho pragmático (Ruiz Gurillo, 2006) como la metáfora o la ironía, se profundiza en las habilidades metapragmáticas que favorecen tanto su creación como su comprensión (Ruiz Gurillo, 2016). De este modo, el punto de vista del hablante/escritor ha de complementarse necesariamente con el del oyente/lector. Con el fin de ilustrar tales aspectos teóricos, se expondrán algunas de las habilidades metapragmáticas que pone en marcha tanto el cómico a la hora de producir monólogos humorísticos como el hablante de la conversación espontánea. El análisis de un género propiamente humorístico, el monólogo, de carácter planificado, público e interaccional se completa con el de un género no humorístico, la conversación espontánea, que es de carácter no planificado, privado y principalmente interactivo. Ello permite ilustrar algunos de los contextos en los que se usa y se infiere humor en español.
\end{abstract}

Palabras clave: humor, metapragmática, adaptabilidad, monólogo, conversación

\begin{abstract}
The main aim of this paper is to analyze the metapragmatic ability of humor in Spanish. Taking into account that it is a pragmatic phenomenon (Ruiz Gurillo, 2006), such as metaphor or irony, this research delves into the metapragmatic abilities that promote not only its creation but also its comprehension (Ruiz Gurillo, 2016). Therefore, the speaker/writer's perspective may be complemented with the listener/reader's point of view. In order to illustrate these theoretical aspects, we will analyze some of the metapragmatic abilities that both comedians in monologues and speakers in conversation carry out while using humor. Firstly, a humorous genre, i.e. the monologue, will be described as a planned, public and interactional discourse. Secondly, a non-humorous genre, i.e. the spontaneous conversation, will be presented as an unplanned, private and interactive discourse. In sum, this research illustrates some contexts in which humor is used and inferenced in Spanish.
\end{abstract}

Keywords: humor, metapragmatics, adaptability, monologue, conversation

‘Este artículo ha sido posible gracias a los Proyectos de Investigación FFI2015-64540-C2-1-P Género, humor e identidad: desarrollo, consolidación y aplicabilidad de mecanismos lingüísticos en español (MINECO-FEDER, UE) y PROMETEO/2016/052 Humor de género: observatorio de la identidad de mujeres y hombres a través del humor (Generalitat Valenciana), así como a la Red Temática en Estudios de Análisis del Discurso (FFI2017-90738-REDT (MINECO-AEI, UE). 


\section{INTRODUCCIÓN}

Este trabajo se centra en el humor entendido como hecho pragmático y como habilidad metapragmática. Siguiendo lo establecido en Ruiz Gurillo (2006), el humor es un hecho pragmático que se explica a la luz de la pragmática, entendida principalmente como perspectiva (Verschueren, 1999). La luz que arroja la pragmática sobre los diversos fenómenos lingüísticos del español nos permite describir un conjunto de aspectos implicados en el humor, a la vez que explicar su funcionamiento.

Los seres humanos disponemos de diversas habilidades que tienen que ver con procesos complejos de metarrepresentación (Wilson, 2000; Ruiz Gurillo, 2008). En estas habilidades interviene tanto el desarrollo del lenguaje como el manejo de diversos aspectos cognitivos. Entre ellas se encuentra la metáfora, la ironía o el humor. En este sentido, el humor es una habilidad metapragmática más de los seres humanos. Compartimos la concepción de Verschueren (1999: 188) de que la metapragmática es "the systematic study of the metalevel where indicators of reflexive awarenesss are to be found in the actual choice-making that constitutes language". La orientación que defendemos conecta con las habilidades metapragmáticas ${ }^{1}$ (Gombert, 1992: 12) y pone el foco de atención tanto en las actividades reflexivas del uso del lenguaje como en las habilidades de los hablantes para monitorizar y planificar sus propios métodos de procesamiento lingüístico para usar y comprender el humor.

El humor es un tipo de comunicación non-bona fide (Raskin, 1985; Attardo y Raskin, 1991; Attardo, 2008; Partington, 2006; Shilikhina, 2017) que se apoya en una incongruencia para generar sus efectos (Attardo, 2008; Archakis y Tsakona, 2005; Ruiz Gurillo, 2012). La incongruencia supone un desajuste entre los dos guiones o marcos que se activan en el humor, lo que, previsiblemente, ocasionará la resolución en favor de uno de ellos o un espacio de mezcla donde se combinan sus informaciones e inferencias (Ruiz Gurillo, en prensa).

Este tipo de comunicación humorística conlleva la adopción de un modo humoristico. En concreto, el hablante/escritor pone en alerta al oyente/lector al indicarle que no está siendo cooperativo ${ }^{2}$. Con esta comunicación activa un escenario lúdi-

\footnotetext{
${ }^{1}$ Ello implica, además, que se ha desarrollado la conciencia metapragmática. Sobre este aspecto pueden consultarse los trabajos de Crespo y Alfaro (2009), Timofeeva (2014), Timofeeva y Ruiz Gurillo (en prensa).

${ }^{2}$ Mientras la comunicación bona fide es cooperativa, la non-bona fide no lo es, de manera que, por un lado, el hablante no está comprometido con la verdad de lo que dice y, por otro, el oyente es consciente de que no existe dicho compromiso (Raskin, 2007: 99). Aunque es evidente que no existe una distinción dicotómica entre la comunicación cooperativa y la no cooperativa (Shilikhina, 2017: 117), el humor, la ironía y el sarcasmo, la mentira/hipocresía y la comunicación absurda constituyen
} 
co, emplea ciertas marcas humorísticas y atenúa su grado de cooperación. Todo ello conlleva un esfuerzo cognitivo mayor por parte del oyente/lector para comprender las inferencias que quiere transmitir. De hecho, la inferencia de que el discurso se inscribe en el modo humorístico es clave para la adecuada comprensión del humor.

Considerando tales aspectos, el humor se aborda, en primer lugar, como habilidad metapragmática (epígrafe 2). Un repaso de los aspectos implicados en la variabilidad, negociabilidad y adaptabilidad constituirá la base sobre la que se analizarán los procesos implicados en dos géneros diferenciados: uno propiamente humorístico, el monólogo (epígrafe 3), y otro no humorístico donde el humor es opcional, aunque esperable (epígrafe 4), la conversación espontánea.

\section{LA HABILIDAD METAPRAGMÁTICA DEL HUMOR}

Como estamos viendo, el humor es una habilidad metapragmática (Ruiz Gurillo, 2016). El hablante/escritor lleva a cabo una continua elección, consciente o inconsciente, donde la variabilidad, la negociabilidad y la adaptabilidad son las nociones clave (Verschueren, 1999). Expondremos a continuación en qué consiste cada una y cómo afecta al uso y a la comprensión del humor.

\subsection{La variabilidad}

La variabilidad alude al rango de posibles elecciones que tiene a su alcance el hablante/escritor. Afecta al contexto en el que se produce el humor, al registro y al código empleado y a la relación entre los participantes, entre otros aspectos. Ello supone que el hablante/escritor ha de observar las posibles variables que le ofrece la lengua y la pragmática. Así por ejemplo, el contexto en el que se produce el humor es importante, pues no es lo mismo hacer un discurso humorístico en un escenario que hacerlo en una conversación con amigos. El contexto, a su vez, repercute en otros aspectos de variabilidad. También cabe considerar las diversas nociones implicadas en el registro (canal, sociolecto, nivel de planificación, dinamismo, inmediatez, tono, tema, etc.) y la relación entre los participantes del acto humorístico (formalidad, conocimiento mutuo, etc.). En nuestra opinión, dos son los aspectos fundamentales que cabe abordar a la hora de entender la variabilidad del humor:

-El grado de planificación. Existen un buen número de géneros propiamente humorísticos (Tsakona, 2017) que llevan asociado un grado alto de planificación.

cuatro tipos de comunicación non bona fide que se situarían en la zona difusa donde no se cumple estrictamente el principio de cooperación. 
Entre otros se encuentra el chiste, la parodia, la comedia de situación o el monólogo humorístico. En concreto, en el monólogo se planifican los ganchos y el remate final del monólogo. Estos son elementos humorísticos integrados en la estructura del texto (Attardo, 2001 y 2008) que permiten observar la incongruencia y, en su caso, resolverla. Además, el buen cómico decide el tipo de temas que va a exponer en el escenario, el tono con el que los va a plantear, o si va a interactuar o no con el público.

En cambio, la conversación espontánea no planifica el humor. En este registro el humor es un rasgo opcional (Tsakona, 2017) aunque, cuando aparece, lo hace como una estrategia conversacional que emplea el hablante. De forma general, el humor aparece en una intervención de uno de los participantes. Esta intervención irónico-humorística puede continuarse o no. Si se continúa, se fomenta el modo humorístico. Si no es así, el humor no es aceptado por el resto de participantes y se produce lo que se conoce como humor fallido (Bell, 2009; Alvarado, 2013 y 2016).

-El grado de publicidad. Los géneros propiamente humorísticos se caracterizan por contar con una audiencia como destinatario (Cortés y Camacho, 2003; Koch y Oesterreicher, 2007). La elaboración de un monólogo supone contar con que será llevado a escena, ya sea en una sala, teatro o set de televisión. Ello implica que, desde el punto de vista de la variabilidad, el cómico ha de practicar su guion y comprobar que funciona.

Por su parte, la conversación es un discurso privado en el que las bromas se hacen entre los participantes. El humor surge de alguna situación graciosa que, por lo común, forma parte del conocimiento común compartido de los interlocutores.

\subsection{La negociabilidad}

La negociabilidad pone en marcha ciertos principios y estrategias flexibles (Verschueren, 1999: 59), de modo que el hablante/escritor negocia las múltiples elecciones que la lengua le ofrece en un contexto determinado: establece una elección dinámica entre los significados explícitos e implícitos, las realza entre los participantes y negocia los estereotipos que se infieren de ellas. Como vimos en Ruiz Gurillo (2016: 89), la negociabilidad conecta con los mecanismos lógicos implicados en el humor, entre los que diferenciamos aquellos basados en razonamientos, como el quiasmo, la yuxtaposición o el paralelismo, o aquellos basados en relaciones sintagmáticas (Attardo, Hempelmann y Di Maio, 2002). También el blanco de la burla que puede afectar a uno o varios participantes de la comunicación.

Desde nuestro punto de vista, la negociabilidad se relaciona estrechamente con la interaccionalidad del discurso humorístico. Pese a la diferencia dicotómica entre discursos monológicos y dialógicos, se viene observando en los últimos tiempos 
que las diversas partes implicadas tanto en la comunicación oral como en la escrita o tecnológica son importantes. Para dar cabida a este hecho se ha desarrollado el concepto de humor interaccional (Chovanec y Tsakona, 2018). En el caso de la conversación espontánea que analizaremos se trata, evidentemente, de una interacción. Por lo que afecta al monólogo humorístico, y como hemos venido defendiendo en diversos trabajos (Ruiz Gurillo, 2013a, 2019a), ciertos monólogos constituyen un diálogo con el público.

\subsection{La adaptabilidad}

Por último, la adaptabilidad supone la modulación dinámica de las elecciones metapragmáticas por medio de los diversos aspectos cognitivos e ideológicos implicados. Los guiones o marcos que se manejan, así como la incongruencia que se deriva de ellos, son aspectos que se adaptan a cada comunicación. Asimismo, la adaptabilidad persigue el logro de fines comunicativos concretos, como divertir a la audiencia, hacer reír o generar un estado de opinión. De hecho, el discurso humorístico puede, entre otras funciones, transmitir el mantenimiento del statu quo, abogar por la subversión de determinados estereotipos, ayudar a afrontar problemas sociales o psicológicos, o favorecer el establecimiento de lazos entre los participantes en el acto comunicativo.

Con el objeto de asumir esta visión metapragmática del humor, se tiene en cuenta el concepto de secuencia humorística. Una secuencia humorística es una unidad estructural y temática que concluye con un gancho (jab line), entendido como enunciado humorístico integrado en la estructura del texto, o con un remate (punch line), si se trata del último enunciado humorístico de dicho discurso (Attardo, 2001 y 2008). Para lograr los fines perseguidos, se emplean ciertas claves que denominamos marcas e indicadores humorísticos. Una marca humorística es un elemento extralingüístico, paralingüístico o lingüístico que ayuda a interpretar el humor. Por ejemplo, ciertos gestos, como sacar la lengua, pueden avisar al interlocutor sobre las intenciones irónicas. Las pausas, la intensidad de la voz o el tono son algunas de las marcas que también colaboran. Por otro lado, los indicadores humorísticos son elementos que se interpretan de manera humorística en un contexto dado. Así, el empleo de polisemia, la paronimia, el cambio de registro (por ejemplo, de informal a formal), el empleo de ciertas unidades fraseológicas, etc., son procedimientos humorísticos usados en contextos humorísticos.

El concepto, que proviene del análisis conversacional (Schegloff, 2007), permite vislumbrar un acomodo del corpus tanto de monólogos como de conversaciones 
al hecho del humor interaccional ${ }^{3}$. A su vez, este concepto se adapta a cada uno de los géneros estudiados, dependiendo, como veremos, de su grado de publicidad y, sobre todo, de su grado de planificación.

\section{EL MONÓLOGO HUMORÍSTICO}

El monólogo humorístico es un género propiamente humorístico, siguiendo la propuesta de Tsakona (2017) antes explicada. Por lo que afecta a la variabilidad, es planificado y público. Se concibe como un género de persona a audiencia (Calsamiglia y Tusón, 1999) que cuenta con un texto escrito previo, pero que se lleva a escena como si no estuviera escrito. Su grado de planificación es alto, si bien pueden verse algunos monólogos cuya intención es desarrollarse de modo improvisado. También cabe señalar que, en líneas generales, fomentan un tono informal y tratan de temas al alcance de cualquier individuo.

En cuanto a la negociabilidad, destacamos en especial el carácter interactivo de ciertos monólogos (Ruiz Gurillo, 2013a). Muchos de ellos cuentan con un grado de interacción con el público, lo que ocasiona que haya cierta retroalimentación y cierto dinamismo entre los participantes. Además, el hecho de que se desarrolle cara a cara y de forma inmediata (aquí y ahora) favorece el empleo de ciertos recursos, como los gestos o ciertos rasgos paralingüísticos, como la intensidad de la voz. De este modo, los significados parecen negociarse en contexto y, pese a tratarse de un discurso eminentemente planificado, parece acercarse a la improvisación de la conversación.

Este hecho se refleja en el concepto de secuencia que manejamos para el monólogo. Se trata de una estructura conversacional en la que el monologuista desarrolla un determinado tema a través de una intervención discontinua (Grupo Val.Es.Co., 2014: 22-23; Pons, 2014). Por lo común, esta intervención es interrumpida por las risas y los aplausos de la audiencia. A menudo, y dependiendo de la retroalimentación con el público, el cómico agrega diversas codas o añadidos (Scarpeta y Spagnolli 2009) que generan, a su vez, nuevas risas y aplausos (Véase también Ruiz Gurillo, 2013b, 2014).

Todos estos aspectos se adaptan para conseguir los fines comunicativos, principalmente divertir a la audiencia. Como técnica general, se construye una premisa seria que se remata con una conclusión humorística. En este proceso intervienen marcas e

${ }^{3}$ Se cuenta con un corpus de monólogos compuestos por los 203 textos de Andreu Buenafuente, extraídos de 3 libros (Digo yo, 2009, Sigo diciendo, 2010, y Hablar es gratis, 2011) a los que se suman diversos monólogos dramatizados de sus programas. También se dispone de los 96 monólogos audiovisuales que Eva Hache interpretó en su calidad de presentadora del programa El Club de la Comedia durante 2011 y 2012. En cuanto a las conversaciones, se han extraído un total de 148 secuencias de 67 conversaciones coloquiales, provenientes del corpus del grupo Val.Es.Co. (www.valesco.es). 
indicadores humorísticos. Además, la secuencia puede servir para reforzar el statu quo, reforzar los estereotipos, mostrar solidaridad con un grupo social concreto o subvertir lo establecido (Ruiz Gurillo, 2019a; Ruiz Gurillo y Linares Bernabéu, en prensa).

Tomemos una secuencia de un monólogo de Eva Hache. La cómica ha hablado previamente de su exnovio hippie y explica que la mejor manera de superar la ruptura es encontrar otro novio mayor que ella:

\section{(1)}

EVA HACHE: mira $\downarrow$ cuando cortas con un hombre así $\downarrow$ con un perroflauta de mierda de estos/ lo mejor lo mejor es para compensar echarte un novio de verdad $\downarrow$ un tío que te cuide y que te saque/ que te saque a comer $\downarrow$ que te saque a bailar $\downarrow$ que te saque veinte años

PÚBLICO: RISAS

EVA HACHE: hay también que tener un poquito de alerta en estos casos porque este tipo de hombres al principio dan mucha garantía pero con el tiempo empiezan a comportarse de un forma digamos extraña/ no sé cómo deciros $\downarrow$ es como si de prontoo se le notaran los años que tiene

PÚBLICO: RISAS

EVA HACHE: ¿mm? antes no salíais de la cama y ahora tampoco pero la colcha no se ha movido del sitio

PÚBLICO: RISAS

EVA HACHE: que de pronto un día te dice ¿por qué no vamos a mi casa a ver una pelicula?/y vas ¿y te puedes creer que acabas viendo la película?

PÚBLICO: RISAS

EVA HACHE: te llevas otro disgusto pero hay que reconocer que es muy fácil acabar una relación ¿ $\mathrm{mm}$ ? sin dramas sin discusiones $\rightarrow$ un buen susto $a$ ah PÚBLICO: RISAS

EVA HACHE: y a otra cosa

PÚBLICO: RISAS

Como vemos en (1), Eva Hache consigue sus propósitos empleando los ganchos humorísticos adecuados y apoyándose en ciertas marcas e indicadores humorísticos. Así, en su primera intervención (discontinua) destaca la polisemia de sacar, que se emplea con las valencias sacar [a alguien] [a hacer algo] y sacar [a alguien] [TIEmpo]. Para hacerlo, Eva Hache gestiona a la perfección su dicción y los diversos recursos que ello implica, como la entonación, la pausa previa a la resolución donde opta por el segundo significado de sacar. También en la cuarta intervención (discontinua) vemos cómo gestiona el gancho a través del discurso representado, en 
concreto usando el discurso directo, marcado en la transcripción en cursiva, para representar la voz de su novio.

Por otro lado, y aunque sabemos que el monólogo está planificado, la secuencia (1) manifiesta algunos fenómenos de improvisación, como cuando Eva Hache alarga las vocales (de prontoo). Aspectos conversacionales como el dinamismo se manifiesta en el empleo de la señal fática ¿mm? en las intervenciones (discontinuas) tercera y quinta. También el estilo narrativo oral del discurso directo que emplea para representar la voz de su novio ("te dice ¿por qué no vamos a mi casa a ver una pelicula?") fomenta una mayor retroalimentación del discurso.

Así pues, la cómica observa la gama de variables que le permite la lengua (registro, formalidad, publicidad, planificación), negocia en el contexto los significados que desea que su audiencia infiera (como la polisemia de sacar). Todo ello le permite adaptar la secuencia: usa una premisa seria ("echarse un novio de verdad") para llegar a la conclusión humorística o gancho ("que te saque veinte años"). Como evidencian las risas del público, Eva Hache logra su objetivo de divertir a la audiencia en esta secuencia. Además, evidencia uno de los estereotipos asociados a la mujer, el hecho de tener novios mucho más mayores. El manejo de este estereotipo le permite llegar al gancho final en las intervenciones (discontinuas) quinta y sexta y resolver que la mejor manera de acabar con una relación así es darle un susto "de muerte". Con ello se refuerza el estereotipo de parejas formadas por una mujer joven y un hombre mayor pero, al tiempo, se ofrece una forma jocosa de afrontarlo.

\section{LA CONVERSACIÓN ESPONTÁNEA}

La conversación espontánea es un género no propiamente humorístico en el que, sin embargo, puede encontrarse el humor (Tsakona, 2017). En cuanto a la variabilidad, la conversación no es planificada y es de carácter privado. Dada su coloquialidad, la conversación es de tono informal, desarrolla temas al alcance de cualquier individuo y cuenta con un tenor interpersonal entre los participantes, es decir, el fin último de la conversación es conversar. Como rasgos de negociabilidad cabe destacar su carácter eminentemente interactivo. Los rasgos de retroalimentación, dinamismo entre los participantes, inmediatez o conversación cara a cara están muy presentes.

Todo ello ocasiona que cuando aparece el humor lo hace en una intervención de un participante que ha decidido cambiar el modo serio por el modo humorístico. Aquí interviene la negociabilidad, ya que el hablante ha de negociar el humor en contexto con su(s) interlocutor(es). Esta intervención humorística, dado el carácter interactivo de la conversación, puede continuarse o no ser respondida. Si no se 
responde de ningún modo, estamos ante humor fallido (Bell, 2009, Alvarado, 2013, 2016). Si se responde, puede mantenerse el Principio de Interrupción Mínima (Eisterhold, Attardo y Boxer, 2006), es decir, interrumpir el modo serio únicamente durante una o dos intervenciones, o puede continuarse el humor, dando como resultado una secuencia con 4, 5, 6,7 o incluso muchas más intervenciones humorísticas. Como hemos observado (Ruiz Gurillo, 2019a), el humor continuado (Attardo, 2019) es uno de los fenómenos mejor representados en el corpus de conversaciones coloquiales del grupo Val.Es.Co. ${ }^{4}$

Este carácter interaccional y de negociabilidad se manifiesta en el concepto de secuencia que manejamos para la conversación. La secuencia humorística es una estructura conversacional compuesta por diversos intercambios o diálogos que se estructura en torno a un mismo tema. Debido al carácter no planificado de este género, el humor aparece como una estrategia más empleada por los hablantes. Resulta habitual que alguno de los participantes produzca una intervención humorística que luego es continuada (o no) por el resto de participantes. Cuando el humor se continúa (Attardo, 2019), se genera una secuencia irónico-humorística en la que destaca el modo humoristico (Raskin, 1985; Shilikhina, 2017; Ruiz Gurillo, 2019b).

Por su parte, los participantes que emplean el humor como estrategia lo adaptan a la estructura conversacional, con el objeto primordial de conseguir la risa de su interlocutor o fomentar el modo humorístico, pero también con el fin de emplear el humor como un gran aliado social. De esta manera, el humor permite en la conversación afianzar los lazos de solidaridad entre los participantes, expresar autohumor, atacar al interlocutor o facilitar un medio de defensa ante los ataques, entre otros aspectos (Lampert y Ervin-Tripp, 1998 y 2006; Hay, 2000; Coates, 2003; Yus, 2016).

En (2) dos hablantes mujeres de menos de 25 años comentan acerca de uno de sus compañeros. Como señala $\mathrm{MJ}$, es mucho mayor que ellas y quiere ligar con todas las chicas. La secuencia de carácter narrativo se sitúa en el modo humorístico, pues las dos participantes colaboran con intervenciones humorísticas, lo que ocasiona que el humor se continúe a lo largo de ocho intervenciones:

MJ: es muy pesao// además de verdad\$

M: $\quad \S$ es un plasta

${ }^{4}$ Este corpus, que se puede consultar en (http://www.valesco.es/?q=corpus) está compuesto por conversaciones coloquiales que cumplen con los rasgos establecidos por el grupo Val.Es.Co. para garantizar la coloquialidad de las muestras, como la toma de turno no predeterminada, la retroalimentación, el dinamismo conversacional, el tema no especializado o la relación de igualdad entre los participantes. 
MJ: es MUY / muy muy pesao/ a(d)emás/ ¡es que no te deja hablar!/// te tiene que contar todas sus historietas amorosas $\rightarrow$ cuando es que se las inventa/ el otro día/ estaba en la cafetería $\downarrow$ estábamos// yy me parece que eraa/ cuando acabábamos un examen $\downarrow$ o no sé qué/ y él estaba en la cafetería/ total quee/ nada// nos sentamos con él y tal $\uparrow / /$ y estábamos allí y se acerca una chica ¿no?/// y hablando hablando oigo como dice laa- laa- la chica esta a él $\uparrow$ / eres/ don FANTASIman [(RISAS $=]$

$\mathrm{M}: \quad[(\mathrm{RISAS})]$

$\mathrm{MJ}:=$ total/ que la chica se va/ y él se queda/ pero con los ojos to's abiertos $\rightarrow$ se viene para allá y dice/ ¿sabéis lo que me acaban de decir?\$

M:

MJ: y yo $\rightarrow$ ¿qué?/ dice que soy don fantasiman/ dice ique tengo mucha fantasía! §

M:

MJ: pero/ pobrecillo/ to(do) deprimido y digo ¡madre mia!/ digo es que es verdads

M:

MJ: $\quad$ \iuna fantasía!/ a una amiga mía $\uparrow$ diciéndole/ noo $\downarrow$ es quee/// tú no sabes lo que es estar conmigoo [ee]

M:

[sí]/ a mí también me lo sueltaa

MJ: espera $\downarrow$ espera/ en una chimeneaa $\rightarrow$ des $N U d o s \rightarrow / /$ junto al calor/del hogar $\rightarrow$ pero bueno $\downarrow$ ¡ASÍ!/ y mi amiga decía pero bueno§

M:

$$
\text { spero TÍO }
$$

MJ: pero este tio/ ipero será guarro!/ ¿ia mi qué me dice!? / si yo paso de él como de la mierda $\downarrow$ tanto es quee/ no§

M: $\quad \$$ a mí me viene y me dice $\uparrow$ / Marial tú tienes ALgo/ que solamente un hombree/como yo/ puede sacar de ti y tú $\uparrow /$ ¡noo! si yo soy una puta mierda $\downarrow$ o seaa $\rightarrow$ no hay nada que buscar dentro/ tú tranquilo/ no te esfuerces $^{5} / / / /$ no sé-no- yo sé que tienes mucho dentro/ y solamente YO/ sabría cómo sacarlo/ y tú ${ }^{\circ}$ (bah) $\% / /$ ¡VENga moDEsto! baja que sube Xavi ${ }^{\circ}$ (baja un poco $)^{\circ} \&$

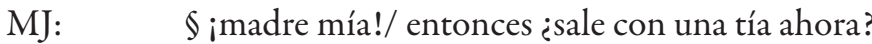

M: ay ¡no sée!

(Conversación [180.B.1], secuencia 143)

Como observamos en (2), se trata de una secuencia humorística inserta en una conversación que no es de carácter humorístico. Por lo que afecta a la variabilidad,

${ }^{5}$ Entre risas. 
el hecho de estar ante un registro no planificado influye sobre el tipo de humor que encontramos. En la mayor parte de las secuencias humorísticas conversacionales el humor no se planifica, sino que surge sobre la marcha. Vemos en (2) que MJ empieza a contar una historia sobre un compañero mayor que ella, historia que comparte con M. Según el relato, una chica se le acercó al mencionado compañero y le llamó fantasiman. La secuencia de historia se desarrolla en modo humorístico, pues $\mathrm{M}$ acepta la historia, al tiempo que colabora en el humor, principalmente con risas. Ello demuestra que reconoce y aprecia el humor (Alvarado, 2013 y 2016). Algunas intervenciones más tarde, $\mathrm{M}$ también aporta su experiencia sobre ese compañero mayor que ellas en edad y sobre cómo intenta ligar con todas.

Evidentemente el blanco de la burla de toda la secuencia es el chico del que se habla. La conversación es de carácter privado, ya que las participantes comparten sus experiencias sobre aquel del que se burlan.

La negociabilidad se observa en determinados rasgos interactivos. Así, el dinamismo entre las participantes, esto es, el intercambio de los papeles de hablante y oyente facilita que sean ambas las que cooperen para construir la historia. Funciona muy bien la retroalimentación entre ambas, pues tras la primera parte donde es MJ la que aporta sus datos, $\mathrm{M}$ comienza a hablar de su experiencia. También facilitan la interacción el hecho de encontrarse cara a cara y aquí y ahora.

Todos estos aspectos se negocian en contexto, ya que encontramos una intervención de $\mathrm{M}$ solapada en parte con la de $\mathrm{MJ}$ y con la que ha intentado iniciar su comentario ("sí a mí también me lo sueltaa"). MJ, en cambio, le pide más tiempo para concluir su relato ("espera $\downarrow$ espera") y tras ello permite que M cuente su experiencia. En este caso, $\mathrm{M}$ comienza contando lo que le ocurrió, pero luego construye una historia fantástica (Kotthoff, 2011) donde habla de lo que previsiblemente le podría haber dicho en esa situación.

Por lo tanto, observamos cómo las hablantes construyen su relato humorístico de manera cooperativa, gracias al grado de formalidad bajo, a la relación de igualdad entre ellas (dos mujeres de menos de 25 años), a su experiencia común compartida (son amigas), el tema no especializado que abordan, y amparadas por rasgos interactivos como el dinamismo conversacional, la retroalimentación, el hecho de encontrarse cara a cara y que la interacción se dé aquí y ahora. El humor es, en este caso, una estrategia de afiliación (Coates, 2003) que sirve para afianzar la solidaridad entre ellas y el afrontamiento de los problemas. De hecho, permite compartir una experiencia presumiblemente desagradable con un hombre mayor que ellas y transformarla, gracias al modo humorístico, en algo divertido.

Con todo ello, el humor se adapta al contexto de la conversación, por lo que observamos un humor no planificado y espontáneo. A veces se encuentran ciertas 
marcas como las pausas y la entonación o la intensidad de la voz (FANTASIman), aspectos que facilitan la comprensión del humor. También se hallan algunos indicadores humorísticos que, frente a lo que ocurre con el monólogo, no han sido planificados previamente. En (2) encontramos el juego polisémico (Fantasimán, fantasía), el empleo de la fórmula conversacional de sorpresa imadre mía! o de ciertos esquemas fraseológicos (Mura, 2019) (baja Modesto que sube Xavi) que permiten evaluar la historia como jocosa y divertida.

\section{Conclusiones}

La adaptabilidad del humor a cada contexto de uso evidencia procedimientos y efectos diferentes. En un género propiamente humorístico como el monólogo, de carácter planificado, público e interactivo, el humor se adapta para lograr los fines comunicativos perseguidos por el cómico. Además de divertir a la audiencia, el monólogo puede servir para mostrar los estereotipos, reforzar el statu quo, mostrar solidaridad con un grupo social o subvertir lo establecido.

En un género no propiamente humorístico como la conversación donde el humor es una estrategia conversacional más, el humor se adapta al registro no planificado, privado y eminentemente interactivo. En el contexto conversacional los participantes negocian si desean mantener el modo humorístico o no hacerlo, lo que puede ocasionar que el humor se continúe a lo largo de una secuencia. Por lo común, el humor no ha sido planificado previamente, por lo que no se evidencian ganchos o remates ni tampoco la estructura de premisa seria/conclusión humorística que se da en el monólogo. Es cierto que algunas marcas, como las pausas o la entonación, colaboran en la comprensión del humor. Sin embargo, los indicadores humorísticos que encontramos, dado su carácter espontáneo, conectan por lo general con aquellos relacionados con el humor prototípico (Ruiz Gurillo, 2012). En concreto, abundan los juegos de palabras, que incluyen como indicadores la polisemia, la homonimia o la paronimia.

La línea de investigación planteada en este trabajo aborda el humor como competencia, pero también como actuación. De hecho, el humor se produce tanto en el monólogo como en la conversación, pero es imprescindible que la audiencia o el interlocutor reaccione a las diversas inferencias del hablante/escritor para poder valorar si el humor ha logrado sus objetivos. Por ello, la metapragmática del humor profundiza en una nueva vía de estudios que no solo tienen en cuenta el humor como competencia (Attardo, 2001), sino que considera aspectos como la apreciación o aceptación del humor, su apoyo o su continuación. Esta nueva vía del humor 
como actuación (Ruiz Gurillo, 2016; Attardo y Raskin, 2017) habrá de recibir nuevas aproximaciones en un futuro no muy lejano.

\section{BIBLIOGRAFÍA}

Alvarado Ortega, M. Belén (2013): «Failed humor in conversational utterances in Spanish: a matter of (im)politeness », in Leonor Ruiz Gurillo and María Belén Alvarado Ortega (eds.), Irony and Humor: From Pragmatics to Discourse, Amsterdam, John Benjamins, pp. 191-217.

Alvarado Ortega, M. Belén (2016): «Variability, adaptability and negotiability in conversational humor: A question of gender », in Leonor Ruiz Gurillo (ed.), Metapragmatics of Humor: Current Research Trends, IVITRA Research in Linguistics and Literature, Amsterdam, John Benjamins, pp. 192-214.

Archakis, Argiris and Villy Tsakona (2005): «Analyzing conversational data in GTVH terms: A new approach to the issue of identity construction via humor», Humor, 18-1, pp. 41-68.

AtTARdo, Salvatore (2001): Humorous texts: A semantic and pragmatic analysis, Berlin, Mouton de Gruyter.

ATTARDO, Salvatore (2008): «A primer for the linguistics of humor » , in Viktor Raskin (ed.), The Primer of Humor Research, Berlin, Mouton de Gruyter, pp. 101-155.

ATtARDo, Salvatore (2019): «Humor and mirth. Emotions, embodied cognition, and sustained humor», in Lachlan J. Mackenzie and Laura Alba-Juez (eds.), Emotion in Discourse, Amsterdam, John Benjamins, pp. 189-211.

ATtARDo, Salvatore and Viktor RASKIN (1991): «Script theory revis(it)ed: Joke similarity and joke representation model», Humor, 4(3-4), pp. 293-347.

AtTARdo, Salvatore and V. RASkin (2017): «Humor and Pragmatics», in Salvatore Attardo (ed.), The Routledge Handbook of Language and Humor, London, Routledge, pp. 174-188.

Attardo, Salvatore, Christian, F. Hempelmann and Sara Di Maio (2002): «Script oppositions and logical mechanisms: Modelling incongruities and their resolutions», Humor, 15-1, pp. 3-46.

BELL, Nancy (2009): «Responses to failed humor», Journal of Pragmatics, 41, pp. $1825-1836$. 
Calsamiglia, Helena y Empar Tusón (1999): Las cosas del decir. Manual de análisis del discurso, Barcelona, Ariel.

Chovanec, Jan and Villy Tsakona (2018): «Investigating the dynamics of humor: Towards a theory of interactional humor», in Jan Chovanec and Villy Tsakona (eds.), The Dynamics of Interactional Humor. Creating and negotiating humor in everyday encounters, Amsterdam, John Benjamins, pp. 1-26.

CoAtes, Jennifer (2003): Men talk: Stories in the making of masculinities, Oxford, Blackwell.

Cortés, Luis y M. ${ }^{a}$ Matilde Camacho (2003): ¿Qué es el análisis del discurso?, Barcelona, Octaedro.

Crespo, Nina y Pedro Alfaro (2009): «La conciencia metapragmática en la edad escolar $\gg$, Boletin de lingüistica, XXI (32), pp. 7-21.

Eisterhold, Jodi, Salvatore AtTardo and Diana Boxer (2006): «Reactions to irony in discourse: evidence for the least disruption principle», Journal of Pragmatics, 38, pp. 1239-1256.

GomberT, Jean Émile (1992): Metalinguistic development, New York, Harverster Wheatsheaf.

Grupo VAL.Es.Co. (2014): «Las unidades del discurso oral. La propuesta Val.Es.Co. de segmentación de la conversación (coloquial) », Estudios de Lingüistica del Español, 35, pp. 13-73.

HAY, Jennifer (2000): «Functions of humor in the conversations of men and women $\gg$, Journal of Pragmatics, 32, pp. 709-742.

Koch, Peter y Wulf Oesterreicher (2007): La lengua hablada en la Romania: español, francés, italiano, Madrid, Gredos.

Kotтhoff, Helga (2011): «Co-creating fantastic pretense scenarios», Paper delivered at 12th International Pragmatics Conference (IPrA Association). (Manchester, 3-8 July 2011).

LAMPERT, Martin and Susan M. ERVIN-TripP (1998): «Exploring paradigms: The study of gender and sense of humor near the end of the $20^{\text {th }}$ century $\gg$, in Willibad Ruch (ed.): The sense of humor: Explorations of a Personality Characteristic, Berlin, Mouton de Gruyter, pp. 231-270.

LAmpert, Martin D. and Susan M. ERVIN-TripP (2006): «Risky laughter: Teasing and self-directed joking among male and female friends», Journal of Pragmatics, 38, pp. 51-72.

Mura, G. Angela (2019): La fraseología del desacuerdo. Los esquemas fraseológicos en español, Sevilla, Editorial Universidad de Sevilla.

Partington, Alan (2006): The linguistics of laughter: A corpus-assisted study of laughter-talk, London, Routledge. 
Pons, Salvador (2014): «Models of Discourse Segmentation in Romance Languages », in Salvador Pons (ed.), Discourse Segmentation in Romance Languages, Amsterdam, John Benjamins, pp. 1-21.

RASKIn, Viktor (1985): Semantic Mechanisms of Humor, Reidel, Dordrecht.

RASKIN, Viktor (2007): «The sense of humor and the truth», in Willibald Ruch (ed.), The sense of humor: Explorations of a personality characteristic, Berlin, Walter de Gruyter, pp. 95-108.

RuIz GuRILlo, Leonor (2006): Hechos pragmáticos del español, Alicante, Universidad de Alicante.

Ruiz Gurillo, Leonor (2008): «Las metarrepresentaciones en el español hablado», Spanish in Context, 5(1), pp. 40-63.

Ruiz Gurillo, Leonor (2012): La lingüistica del bumor en español, Madrid, Arco/ Libros.

RuIz GURILlo, Leonor (2013a): «El monólogo humorístico como tipo de discurso. El dinamismo de los rasgos primarios», Cuadernos Aispi, (2), pp. 195-218.

Ruiz GURILlo, Leonor (2013b): «Narrative strategies in Buenafuente’s humorous monologues », in Leonor Ruiz-Gurillo and M. ${ }^{a}$ Belén Alvarado-Ortega (eds.), Irony and Humor: From Pragmatics to Discourse, Amsterdam, John Benjamins, pp. 107-140.

Ruiz Gurillo, Leonor (2014): «Infiriendo el humor. Un modelo de análisis para el español», Revista CLAC (Círculo de Lingüistica Aplicada a la Comunicación), 59, pp. 148-162.

Ruiz Gurillo, Leonor (2016): «Metapragmatics of humor: Variability, negotiability and adaptability in humorous monologues», in Leonor Ruiz-Gurillo (ed.), Metapragmatics of humor: Current Research Trends, Amsterdam: John Benjamins, pp. 81-104.

Ruiz Gurillo, Leonor (2019a): Humor de género. Del texto a la identidad en espanol, Madrid, Iberoamericana Vervuert.

Ruiz Gurillo, Leonor (2019b): «En modo humorístico: habilidades metapragmáticas en discursos de humor», en Antonio Briz, M. José Martínez Alcalde, Nieves Mendizábal, Mara Fuertes Gutiérrez, José Luis Blas, Margarita Porcar (coords.), Estudios lingüisticos en homenaje a Emilio Ridruejo (2 vol.), Valencia, Universitat de València, pp. 1231-1242.

RuIZ GuRILlo, Leonor (en prensa): «Humor e ironía», en M. ${ }^{a}$ Victoria Escandell Vidal, José Amenós Pons y Aoife Ahern (eds.), Manual de pragmática, Madrid, Akal.

Ruiz Gurillo, Leonor and Esther LinAREs BernabÉu (en prensa): «Subversive humor in Stand-up comedy: Two case studies in Spanish», HUMOR: International Journal of Humor Research. 
Scarpetta, Fabiola and Anna Spagnolli (2009): «The interactional context of humor in stand-up comedy», Research on Language and Social Interaction, 42.3, pp. 210-230.

SCHegloff, Emanuel A. (2007): Sequence organization in interaction: A primer in conversation analysis, Cambridge, CUP.

Shilikhina, Ksenia (2017): «Metapragmatic markers of the bona fide and non-bona fide modes of communication», in Wladyslaw Chlopicki and Dorota Brzozowska (eds.), Humorous Discourse, Amsterdam, Mouton de Gruyter, pp. 107-130.

Timofeeva Timofeev, Larissa (2014): «El humor verbal en niños de educación primaria: desarrollo de la conciencia metapragmática», Feminismo/s, 24, pp. 195-219.

Timofeeva Timofeev, Larissa y Leonor Ruiz Gurillo (en prensa): «Marcas e indicadores del humor en narraciones escritas de niños y niñas de 8 a 12 años», Spanish in Context.

Tsakona, Villy (2017): «Genres of humor», in Salvatore Attardo (ed.), The Routledge Handbook of Language and Humour, Routledge, Taylor and Francis, pp. 489-503.

Verschueren, Jef (1999): Understanding Pragmatics, London, Arnold.

Wilson, Deirdre (2000): «Metarepresentation in linguistic communication», in Dan Sperber (ed.), Metarepresentations. A multidisciplinary perspective, Oxford, University Press, pp. 411-448.

Yus, Francisco (2016): Humour and Relevance, Amsterdam, John Benjamins.

Fecha de recepción: 28 de mayo de 2019

Fecha de aceptacion: 11 de octubre de 2019 